\title{
Downward Causation in Fluid Convection
}

\author{
Robert C. Bishop \\ Department of Philosophy, MS 14 \\ Rice University \\ Houston, TX 77251-1892 USA
}

To appear in Synthese

\begin{abstract}
Recent developments in nonlinear dynamics have found wide application in many areas of science from physics to neuroscience. Nonlinear phenomena such as feedback loops, inter-level relations, wholes constraining and modifying the behavior of their parts, and memory effects are interesting candidates for emergence and downward causation. Rayleigh-Bénard convection is an example of a nonlinear system that, I suggest, yields important insights for metaphysics and philosophy of science. In this paper I propose convection as a model for downward causation in classical mechanics, far more robust and less speculative than the examples typically provided in the philosophy of mind literature. Although the physics of Rayleigh-Bénard convection is quite complicated, this model provides a much more realistic and concrete example for examining various assumptions and arguments found in emergence and philosophy of mind debates. After reviewing some key concepts of nonlinear dynamics, complex systems and the basic physics of Rayleigh-Bénard convection, I begin that examination here by (1) assessing a recently proposed definition for emergence and downward causation, (2) discussing some typical objections to downward causation and (3) comparing this model with Sperry's examples.
\end{abstract}


The aim of science is not things themselves, as the dogmatists in their simplicity imagine, but the relations among things; outside these relations there is no reality knowable.

Poincaré

\section{Introduction}

Nonlinear phenomena are found in almost all areas of science. Examples include fluid flows, meteorology, plasma physics, geophysics, oceanography, friction and the breaking of metal structures, coupled oscillators, chemical reactions, cell differentiation, neural functions, and almost all instances of pattern formation. Such phenomena are studied utilizing the mathematics of nonlinear dynamics and complex systems theory and are among some of the most difficult mathematical challenges facing scientists.

Although there has been some discussion of nonlinear dynamics and related areas such as chaos, complexity and self-organization in the philosophy of science literature (e.g., Earman 1986; Stone 1989; Hobbs 1991; Batterman 1993; Kellert 1993; Auyang 1998; Smith 1998; Bishop and Kronz 1999), the question of downward causation in such contexts has received much less attention (Juarrero 1999; Scott 1999; Silberstein and McGeever 1999 and are notable exceptions).

Nonlinear phenomena such as feedback loops, inter-level relations, wholes constraining and modifying the behavior of their parts, and memory effects (e.g., hysteresis) are interesting candidates for downward causation. Downward causation has been considered suspicious, however, as possibly violating the causal closure of the physical or as perhaps involving absurdities (e.g., Kim 1999, 28-31).

One of the problems with discussing downward causation has been the lack of precise definitions for such concepts as emergence and downward causation. Typically debate has focused on examples such as a wheel rolling down a hill (e.g., Sperry 1969). Recently Thompson and Varela $(2001,420)$ proposed a definition of emergence incorporating downward causation that goes some way toward achieving more precision:

(TV) A network, $N$, of interrelated components exhibits an emergent process, $E$, with emergent properties, $P$, if and only if:

(a) $\quad E$ is a global process that instantiates $P$ and arises from the nonlinear dynamics, $D$, of the local interactions of $N$ 's components.

(b) $E$ and $P$ have global-to-local ('downward') determinative influence on the dynamics $D$ of the components of $N$.

And (possibly):

(c) $E$ and $P$ are not exhaustively determined by the intrinsic properties of the components of $N$, that is, they exhibit 'relational holism.'

On this proposal, a property does not just emerge qua property; rather, it is instantiated in a 
process or some other dynamical 'entity' unfolding in time.

The goal of this essay is to draw out some new and important insights for metaphysics and philosophy of science from Rayleigh-Bénard convection. I propose convection as a model for downward causation in classical mechanics, far more robust and less speculative than the dubiously obscure examples characterizing many of the debates. Section 2 presents a brief overview of some key concepts of nonlinear dynamics and complex systems. Section 3 introduces the basic physics of Rayleigh-Bénard convection. Section 4 assesses TV for this model. Some typical objections to downward causation are discussed, and the case of RayleighBénard convection is compared Sperry's examples. Concluding remarks in section 5 draw the essay to a close.

\section{Nonlinear Dynamics}

2.1 Nonlinearity. A dynamical system is characterized as linear or nonlinear depending on the nature of the equations of motion describing the system. A differential equation system $d \boldsymbol{x} / d t=$ $\boldsymbol{F} \boldsymbol{x}$ for a set of variables $\boldsymbol{x}=x_{1}, x_{2}, \ldots, x_{n}$ is linear if the matrix of coefficients $\boldsymbol{F}$ does not contain any of the variables $\boldsymbol{x}$ or functions of them; otherwise it is nonlinear. Roughly, a system behaves linearly if any multiplicative change of its initial data by a factor $b$ implies a multiplicative change of its output by $b$.

A linear system can be straightforwardly decomposed into and composed by subsystems. For example linear (harmonic) vibrations of a string can be analyzed as a superposition of normal modes, which can be treated as uncoupled individual subsystems or parts. Basically this is the principle of linear superposition. The composition of the system is then analogous to aggregating these parts ("the whole is the sum of its parts"). The linear behavior of the system in such cases is sometimes called resultant (as opposed to emergent). ${ }^{1}$

In nonlinear systems, by contrast, such a straightforward idea of composition fails. When the behaviors of the constituents of a system are highly coherent and correlated, the system cannot be treated even approximately as a collection of uncoupled individual parts ("the whole is different than the sum of its parts"). Rather, the principle of linear superposition fails and some particular global or nonlocal description ${ }^{2}$ is required taking into account that individual constituents cannot be fully characterized without reference to larger-scale structures of the system. For example, Rayleigh-Bénard convection exhibits what is called generalized rigidity, meaning that the individual constituents are so correlated with all other constituents that no constituent of the system can be changed except by applying some change to the system as a whole. Such holistic behaviors are often referred to as emergent (as opposed to resultant).

The tight coupling between constituents in nonlinear systems is related to the nonseparability of the Hamiltonian, a function which corresponds to the total energy of the system and is related to its time evolution. Roughly, a Hamiltonian is separable just in case there exists a transformation carrying the Hamiltonian describing a system of $N$ coupled constituents into $N$ equations each describing the behavior of one constituent of the system. Otherwise, the Hamiltonian is nonseparable and the interactions within the system cannot be decomposed into interactions among only the individual components of the system. 
2.2 Complex Systems. Several formal definitions of complexity have been proposed for characterizing random, chaotic and other forms of complex behavior, each having various strengths and weaknesses (Grassberger 1989; Wackerbauer et al. 1994). Unfortunately such characterizations of complexity often provide no intuitive access to the issues of emergence and causation at work in complex systems. Typical definitions of complexity are formalized in terms of probabilities with no explicit reference to physical system variables. ${ }^{3}$

Often complex systems are characterized more phenomenologically, involving the following features:

- Many-body systems. There is a large number of constituents, where 'large' refers to the number of constituents necessary for the behavior to be exhibited. Some systems exhibit complex behavior with as few as three constituents.

- $\quad$ Broken symmetry. Spatial symmetries (e.g., homogeneity) may exist before a critical point, but not beyond.

- Hierarchy. There are a number of distinguishable, interdependent levels or nested structures usually requiring different descriptions at the different levels.

- Irreversibility. Such hierarchies are typically associated with irreversible processes.

- Relations. The constituents are not mere aggregates like sand grain piles, but are coupled to each other via some kinds of relations.

- $\quad$ Situatedness. The properties of the constituents depend upon the structures in which they are embedded as well as the environment of the system as a whole.

- Integrity. Systems as a whole display an organic unity of function which is absent if one of the constituents or internal structures is absent or if coordination among the structures and constituents is lacking.

- Integration. There are several components tightly interconnected through feedback loops and other forms of structural/functional relations crucial to maintaining the integrity of the system.

- Intricate behavior. Systems exhibit behavior situated somewhere between simple order and total disorder.

- $\quad$ Stability. The organizational, relational unity of the system is resilient under small perturbations and adaptive under moderate changes in its environment.

- Observer relativity. The complexity of systems depends on how we observe and describe them (Grassberger 1989; Crutchfield 1994).

This list is not exhaustive, but indicates qualitative features arising from theory and experiments on complex physical systems such as Rayleigh-Bénard convection (e.g., Cross and Hohenberg 1993).

2.3 Control Hierarchies and Constraints. The notion of hierarchy in the context of complex systems requires further elaboration. Some systems are comprised of distinguishable levels of structure, where the ordering into hierarchical levels is due to the hierarchy of physical forces and dynamical time scales (e.g., elementary particles, molecules, crystals, solids). In some cases the lower-level constituents may provide both necessary and sufficient conditions for the existence and behavior of the higher-level structures. 
In complex systems, however, levels of structure are often only distinguishable in terms of dynamical time scales and are coupled to each other in such a way that at least some of the higher-level structures are not fully determined by, and even influence and constrain the behavior of constituents in lower-level structures. That is, the lower-level constituents provide necessary but not sufficient conditions for the existence and behavior of some of the higher-level structures (cf. Bishop 2006b; Bishop and Atmanspacher submitted). Furthermore, the lower-level constituents may not even provide necessary and sufficient conditions for their own behavior if the higher-level constituents can influence the behavior of lower-level constituents. This latter kind of hierarchy is called a control hierarchy in order to distinguish such cases from merely hierarchical structure like sand grain plies (Pattee 1973, 75-9; Primas 1983, 314-23).

The control exercised in complex systems takes place through constraints. The interesting types of constraints must actively change the rate of reactions or other processes of constituents relative to the unconstrained situation (e.g., switches and catalysts). Furthermore, constraints must control constituents without removing all their configurational degrees of freedom in contrast, for example, to the case of simple crystals. Such constraints may be external, due to the environment interacting with the system, and/or internal, arising within the system due to the collective effects of its constituents or some other higher-level structural feature. The latter kinds of internal constraints are of primary interest here. ${ }^{4}$

\section{Rayleigh-Bénard Convection}

The fluid equations governing a Rayleigh-Bénard system are the heat transport equation,

$$
\frac{\partial T}{\partial t}+(\mathbf{v} \cdot \nabla) T=\kappa \nabla^{2} T
$$

the Navier-Stokes equation,

$$
\frac{\partial \mathbf{v}}{\partial t}+(\mathbf{v} \cdot \nabla) \mathbf{v}=-\frac{1}{\rho} \nabla p+v \nabla^{2} \mathbf{v}+\boldsymbol{g}
$$

and the continuity equation (conservation of mass),

$$
\frac{\partial \rho}{\partial t}+\mathbf{v} \cdot \nabla \rho+\rho \nabla \cdot \mathbf{v}=0
$$

In (1)-(3), $T$ is the temperature, $\mathbf{v}$ is the velocity, $\rho$ is the density, $\kappa$ is the thermal diffusivity, $v$ is the kinematic viscosity and $\boldsymbol{g}$ is gravity. The velocity, temperature and density are coupled, the fluid flow inducing temperature variations, while changes in fluid density due to temperature induce changes in the fluid flow. Except for a fluid initially at rest, where the initial pressure $p_{0}$ is related to $\rho$ through $\nabla p_{0}=\rho \mathrm{g}$, the pressure is determined implicitly and, hence, is not an independent variable. Note that this means the pressure is not a dynamic variable. For an incompressible fluid, such as water, the pressure is not fixed locally but depends on distant parts of the fluid dynamics (e.g., Paul et al. 2003).

\subsection{Some System-Wide Properties of Fluids. Before discussing Rayleigh-Bénard convection}


proper, I want to provide some context by quickly reviewing a few basic properties of fluids involved in various global or system-wide interactions in fluid dynamics. As Batchelor points out $(1967,2)$, the cohesive force among molecules is long-ranged relative to molecular length scales, falling off as $r^{-7}$ in the case of nonpolar molecules (where the overall molecular charge is neutral), although it can be larger for polar molecules (e.g., Feynman et al. 1963, 12-6). This force provides a means for large collections of molecules to interact with each other through continuous complex interactions of all the electrons in one molecule with all the electrons in several other molecules, not merely through nearest neighbor interactions. This continuous interaction of a molecule with the cohesive forces of several other molecules is a distinguishing features of liquids in stark contrast to an ideal gas, where molecules are roughly dynamically independent (Batchelor 1967, 53).

Although the basis for why fluid elements cohere, cohesive forces are usually ignored in detailed treatments of fluid dynamics as they are relatively weak. Instead such forces form the background for those treatments in the following way. Cohesive forces in fluids cause the molecules to be packed as closely together as repulsive forces of quantum origin allow. This means that when fluid dynamicists speak of 'fluid particles' or 'fluid molecules,' they do not mean individual chemical molecules (e.g., an individual $\mathrm{H}_{2} \mathrm{O}$ molecule in a stream of water). Rather, these 'particles' or 'molecules' refer to collections of roughly $10^{22}-10^{23}$ chemical molecules. In order to avoid terminological confusion, I will refer to these collections as fluid elements, volumes of a large number of chemical molecules that are small when compared with the size of the fluid system, but large when compared with the size of individual chemical molecules. Cohesive forces play a major role in defining the size and contents of these fluid elements.

Considering a fluid at the level of fluid elements motivates what is known as the continuum hypothesis: even though properties such as the mass or velocity of a fluid are ultimately concentrated in the nuclei of individual chemical molecules, and, hence, are not "smeared" uniformly over the volume of the fluid system, nevertheless such quantities can be treated as if they are continuous and can be regarded as being spread uniformly over the volume of a fluid element (Batchelor 1967, 4). This assumption turns out to be well justified (Batchelor 1967; Mareschal 1997). One can then define notions of local velocity, mass and such, where 'local' refers to individual fluid elements.

Moreover, there are two basic kinds of forces which act on fluids. The first group are long-range forces-such as gravity ${ }^{5}$, electromagnetism (when fluids also carry a charge), so-called fictitious forces like centrifugal forces (when fluid motion takes place with respect to an accelerating frame)-called body or volume forces. Such forces act on all elements of a fluid, such that these forces act equally on all matter within a given volume of fluid. The second group are short-range forces of molecular origin and are appreciable only at distances on the order of the separation of molecules in the fluid. These latter forces are called surface forces because they mainly act on a thin layer adjacent to the boundary of a fluid element, where the thickness of the layer is equal to the depth the forces penetrate. Examples of such forces would be contact forces due to momentum transport across a common boundary between two fluid elements or forces arising from short-range interactions among molecules on the two sides of the common boundary. 
Both kinds of forces are taken into account in the hydrodynamic equations above such that the dynamics of fluids is not merely that of nearest neighbor interactions among fluid elements. Furthermore, the volume and surface forces as well as their distribution throughout a fluid system are modified by the motions of fluid elements relative to each other.

Spatial correlations also play an important long-range role in the dynamics of fluid systems. The positions of fluid elements are not arbitrary given the fact that one element occupies some position centered around $\boldsymbol{r}_{1}$ prevents all other fluid elements from occupying that position. Due to fluid element interactions and the symmetry properties of the state vectors describing their positions, different values of the relative position $\left(\boldsymbol{r}_{2}-\boldsymbol{r}_{1}\right)$ between two fluid elements do not appear with equal likelihood, hence the simultaneous positions $\boldsymbol{r}_{1}$ and $\boldsymbol{r}_{2}$ of the two elements are spatially correlated. It turns out that there is a simple relationship between the spatial integral of the correlation function representing spatial correlation and the mean square fluctuation of the density of elements composing a fluid (Pathria 1972, 447-50), meaning the spatial distribution of the elements is influenced by the presence of such correlations. This coupling due to correlations leads to collective behavior, contributing to the formation of coherent structures. When all fluid elements become so highly correlated with each other that the behavior of individual elements can be modified only by global changes (e.g., by changing the temperature gradient of the system), the system exhibits generalized rigidity. ${ }^{6}$

An important point regarding fluid systems is that there is a discernible hierarchy related to the kinds of dynamics taking place at different time scales. Focusing on (2), the $v \nabla^{2} \mathbf{v}$ term represents diffusive processes associated with the dynamics of individual fluid elements and their exchange of chemical molecules, while the $(\mathbf{v} \cdot \nabla) \mathbf{v}$ term represents convective processes associated with large scale fluid motions and structures. The Reynolds number is a dimensionless parameter that roughly characterizes the effectiveness of convective versus diffusive processes. For small values, the slower time scale (shorter length scale) diffusive processes are dominant, while at high values the faster time scale (longer length scale) convective processes are dominant. The interplay between these two sets of time and length scales is important. If the values of the Reynolds number is too small, coherent long-lived structures typically do not form. On the other hand, if the value is too large, turbulence dominates the flow and coherent shortlived structures only form intermittently. It is in a range in between the extremes where coherent long-lived structures form.

Lastly, I briefly mention that the velocity-time autocorrelation function has a long-time tail or memory effect (first observed by Alder and Wainwright 1970): through various interactions, a fluid element exchanges momentum with its surrounding neighbors, which, in turn, is transmitted throughout the fluid system (Pameau and Résibois 1975; Mareschal 1997, 317-8). This means that information about the motion of an individual fluid element is communicated to other elements throughout the system over some time scale characteristic of the system.

3.2 Rayleigh-Bénard Convection. Rayleigh-Bénard convection provides a concrete example of a complex nonlinear system. Initially a layer of fluid is sandwiched between two horizontal thermally conducting plates. The lower plate is heated while the upper one is maintained at a fixed temperature, establishing a temperature gradient $\Delta T$ in the vertical direction. The fluid near 
the lower plate undergoes thermal expansion and is less dense than that above, creating an instability at a finite wavelength in the presence of gravity. This buoyancy force tends to lift the whole mass of fluid from the lower plate (a consequence of the conservation of mass), while the upper plate acts as an external constraint against such motion. As long as $\Delta T$ remains small enough, thermal conduction dissipates the temperature gradient sufficiently to maintain a stabile state such that the fluid near the lower plate does not rise. In this state, the energy lost by the system due to dissipation balances the amount of potential energy available to be released by the system due to the density gradient in the direction opposite to gravity. ${ }^{7}$

To maintain this subtle balance, however, system boundaries and symmetries as well as conservation laws also play an important role acting as system-wide, global constraints on the motion of fluid elements (e.g., Busse 1978, 1932-6). The system as a whole establishes the allowable states of motion accessible to fluid elements. If there were no such constraints, the fluid in the uniform state could in principle flow in an arbitrary number of directions and an infinite number of convection patterns would be possible. For example, conservation of mass imposes restrictions on the fluid velocity (Batchelor 1967, 73-5), while system geometries and symmetries strongly influence fluid flows and allowable pattern formation (Cross and Hohenberg 1993).

In this globally stable conductive state, local variations in fluid density are the seeds leading to large-scale pattern formation. Typically these density variations are relatively small, but when $\Delta T$ passes a critical value $\Delta T_{c}$, the system becomes globally unstable and sensitive to small perturbations in fluid density. Now more potential energy is being released due to the temperature gradient than can be dissipated, and the relevant dynamics shifts from the slower diffusive timescale (small-scale effects) to the much faster convective timescale (largescale effects). This extra energy drives instabilities ${ }^{8}$ leading to a breakup of the stable conductive state and, eventually, to large-scale rotating structures resembling a series of parallel cylinders called Bénard cells, the simplest case of Rayleigh-Bénard convection (Figure 1). Associated with this new stable pattern is a large-scale, global constraint on the individual

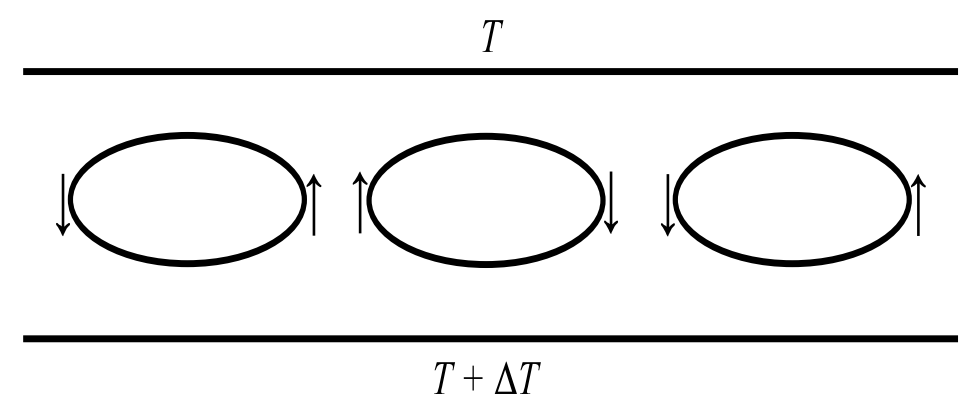
motions of fluid elements due to a balancing among effects owing to the structural relations of each fluid element to all other fluid elements, system boundaries and symmetries, and conservation laws.

If $\Delta T$ is increased beyond a second threshold $\Delta T_{\alpha}$, the cells begin to oscillate transversely 
in complicated ways. Further increases in $\Delta T$ lead to destruction of the cells and to chaotic behavior in the fluid, where spatial correlations persist. When $\Delta T$ is sufficiently large, fluid motion becomes turbulent and uncorrelated at any two points in the fluid.

Rayleigh-Bénard convection under increasing $\Delta T$ exhibits typical phenomena associated with nonlinear systems such as period-doubling cascades, phase locking between distinct oscillatory modes and sensitive dependence on initial conditions. For small aspect ratios (ratio of plate separation to plate length), the critical dynamics is characterized by a strange attractor of less than five dimensions in state space. Since a fluid continuum has an infinite number of dynamical variables in general, the fact that so few control the dynamical behavior of RayleighBénard convection is remarkable. For large aspect ratios, the number of degrees of freedom involved appears to increase greatly.

The Rayleigh-Bénard system clearly exhibits the features listed in $\S 2.2$. As $\Delta T$ exceeds $\Delta T_{c}$, the homogeneity of the distribution of the fluid elements and some of the spatial symmetries of the container are broken and the fluid elements self-organize into distinguishable Bénard cells. There is a hierarchy distinguished by dynamical time scales (molecules, fluid elements, Bénard cells) with complex interactions taking place among the different levels. Fluid elements are situated in that they participate in particular Bénard cells within the confines of the container walls. The system as a whole displays integrity as the constituents of various hierarchic levels exhibit highly coordinated, cohesive behavior. Additionally, the organizational unity of the system is stable to small perturbations in temperature and adapts to larger changes within a particular range.

Furthermore, Bénard cells act as a control hierarchy, constraining the motion of fluid elements. Bénard cells emerge out of the motion of fluid elements as $\Delta T$ exceeds $\Delta T_{c}$, but these large-scale structures determine modifications of the configurational degrees of freedom of fluid elements such that some motions possible in the equilibrium state are no longer available. In the original uniform state, the fluid elements can exhibit a particular range of motions constrained by the system symmetries and boundaries, by body forces such as gravity, and so forth. In particular, as long as $\Delta T<\Delta T_{c}$, fluid elements cannot access rotational states of motion characteristic of Rayleigh-Bénard convection. In the new nonequilibrium steady state, the fluid elements exhibit coherent motion (Bénard cells), but most of the states of motion characteristic of the original uniform state are no longer accessible (e.g., fluid elements cannot sit motionless). It can be shown that the uniform and damped modes follow-or are enslaved by-the unstable growing mode leading to cell formation. Basically, the variables characterizing steady and damped modes come to be systematically replaced by variables characterizing the growing mode; consequently there is a corresponding reduction in the degrees of freedom characterizing the system's behavior (e.g., Haken 1983a, chapters 7-8; 1983b, chapter 7). Such a reduction of the degrees of freedom is crucial to the large-scale behavior of convection systems (Greenside, et al. 1982; Malraison, et al. 1983). ${ }^{9}$

There is an alternative analysis of the development of the instability, where the fluid velocity is locked onto the evolution of the temperature field. Under reasonable conditions, the timescale is governed by the diffusion of the temperature (a nonlinear process) and the velocity field responds instantaneously to changes in the fluid density induced by the temperature field (e.g., Guyon et al. 2001, chapter 10). Changes in the fluid velocity then act back on and drive the 
temperature field.

\section{Downward Causation and Rayleigh-Bénard Convection.}

How are we to understand the causal interplay between fluid elements and Bénard cells? The fluid elements are necessary for the existence of Bénard cells (no fluid elements, no cells). But the dynamics of fluid elements themselves are not sufficient on their own to bring about such structure; temperature gradients, gravity, long-range forces and correlations, physical boundaries and symmetries, conservation laws among other things are also involved (recall the degeneracies in fluid motion mentioned earlier). Context, then, plays a crucial role in pattern formation. Indeed, in the absence of gravity, Rayleigh-Bénard convection would never arise because there would be no buoyancy force. Cell formation means the fluid elements are correlated with each other through myriad physical relations ${ }^{10}$, reflecting large-scale structural constraints on the dynamics of the fluid elements through these emergent relations (e.g., enslavement, bulk flow ${ }^{11}$ ). Bénard cells even modulate their fluid element and energy intake to maintain cohesion within a range of variations in $\Delta T$.

The relationship between fluid elements and cells is not a simple matter of a feedback mechanism, at least during the process of pattern formation. A feedback mechanism typically involves a reference state and a number of feedback-regulated set points so that comparisons between the evolving and reference states can be made and adjustments effected (e.g., a thermostat). But during pattern formation in Rayleigh-Bénard convection the appropriate reference states do not exist: The original uniform state is destroyed and a new nonequilibrium steady state forms (Bénard cells). This new steady state can function as a new reference state, but it is at the level of large-scale structure, where self-regulation of the cells takes place (e.g., responses to changes in $\Delta T$ ), further modifying the behavior of the fluid elements.

The fact that all fluid elements in the nonequilibrium state are correlated with each other indicates that these constraints on the fluid elements' states of motion is enforced globally (i.e., beyond mere nearest neighbor interactions). One mechanism through which this arises is stable bulk shear flow of the Bénard cells acting on fluid elements. Shear flow occurs when adjacent layers of fluid slide over one another. So, for example, different layers of Bénard cells rotate differentially-inner layers rotate faster than outer layers-about the cell center in a steady bulk shear flow. Such steady shear flow acts to constrain/modify the dynamics of fluid elements in the following way. Consider a fluid eddy, a coherently rotating system of fluid elements. The eddy is characterized by the distance over which it remains coherent, the eddy coherence length $l_{e}$, which is roughly the distance between two adjacent eddies of comparable scale. In the presence of a random background flow, fluid elements of a given eddy, which move a distance $l_{e}$, are subjected to the effects of adjacent eddies and are no longer identifiable with their original eddy. In other words the original eddy will decohere-the coherently rotating fluid elements will break up-as the fluid elements originally associated with it are carried away by other eddies (a form of the process know as advection). This process takes place over a time scale known as the eddy lifetime, $t_{e}$. This loss of correlation arises because the random background flow acts both to scramble the original eddy's motion and to spread the latter's energy from one scale to another (generally cascading downward to smaller scales). Such processes can lead to the rapid 
destruction of large-scale structures, or prevent such structures from forming, and play an important role in turbulence.

In the presence of a stable bulk shear flow, however, $t_{e}-$ a measure of the eddy's coherence time-is modified because the background shear flow tends to stretch the eddy due to the fact that its different fluid elements are carried along at different speeds by the shear flow. In effect, $t_{e}$ is lengthened as the advective effects of other eddies and the destructive effects of random flows are suppressed by the larger-scale shear flow. Those elements of fluid flow that are random get carried along with and enslaved by the bulk shear flow before disruption of local- and medium-scale patterns can occur. ${ }^{12}$

This is an important process by which Bénard cells maintain themselves and limit the range of motions accessible to fluid elements. Such stability is due to the collective effects of steady, large-scale, shear fluid motion suppressing local deviations. So although the fluid elements are necessary to the existence and dynamics of Bénard cells, they are not sufficient to determine that dynamics, nor are they sufficient to fully determine their own motions. Rather, the large-scale structure supplies a governing influence constraining the local dynamics of the fluid elements (again, recall the fluid degeneracies and their curtailment discussed previously). ${ }^{13}$ The fluid elements, the large-scale structures and the system-wide forces and symmetries together form necessary and sufficient conditions for the behavior of the convection system and its constituents. Note that this influence does not involve qualitatively new forces, but the collective effects of the action of the Bénard cells on the fluid elements. ${ }^{14}$

4.1 The Proposal by Thompson and Varela. Element (a) in TV reads " $E$ is a global process that instantiates $P$ and arises from the nonlinear dynamics, $D$, of the local interactions of $N$ 's components." The term "global process" here should be understood in a relationally dynamic sense, meaning that the process $E$ arises from the interactions of the components and functions as a (quasi)stable unit, but what acts as a unit is strongly dependent on the relations that develop among the components of $N$ (the evolutionary history and context play a key role here). The relations that develop among the components are driven by the dynamical evolution such that multiple possibilities for various processes to be manifested exist (e.g., different possible convection patterns in Rayleigh-Bénard Convection). ${ }^{15}$

Referring only to "nonlinear dynamics" in (a) may also be insufficient as it appears that more needs to be specified. The couplings among system constituents as suggested by many of the features characterizing complex systems (\$2.2)-implying that nonseparability of the Hamiltonian may be an important issue (cf., Kronz 1998)-as well as the values of system parameters are crucial to pattern formation. In addition, external constraints play an important role in the formation and maintenance of Bénard cells, so these must be part of the characterization of the processes instantiating emergent properties. Emphasizing the contextual features of the dynamics is also important.

Bénard cells arise from the dynamics of fluid elements in the Rayleigh-Bénard system as $\Delta T$ exceeds $\Delta T_{c}$, where each fluid element becomes coupled with every other fluid element (element (a) of TV). More importantly, Bénard cells act as a control hierarchy, constraining and modifying the trajectories of fluid elements; that is, Bénard cells have a "determinative influence" on the dynamics of the fluid elements as lower-level system components (element (b) 
of TV). Moreover, although the fluid elements are necessary for the existence of Bénard cells, the former are insufficient to totally determine the behavior of the latter. This relationship between necessary and sufficient conditions seems implicit in (b), but due to its importance in issues surrounding reduction and emergence, this relationship should be brought out explicitly (e.g., Bishop 2006b; Bishop and Atmanspacher submitted).

This relationship is a nonquantum kind of "relational holism" understood in Teller's (1986) sense, where, the relations among constituents are not determined solely by the constituents' intrinsic properties. The properties of integrity, integration and stability exhibited by Bénard cells are relationally dynamic properties involving the nonlocal relation of all fluid elements to each other (element (c) of TV). Such global interactions along with evolutionary history and context all play a role in the determination of which convection patterns arise; the intrinsic properties of fluid elements alone plus the force laws underdetermine these outcomes. However, the behavior of Bénard cells as units differs from holistic entanglement in quantum mechanics in the sense that fluid elements may be distinguished from each other while they are simultaneously identified as members of particular Bénard cells and participate in interaction with fluid elements throughout the system. In this context focusing on the nonseparability of the Hamiltonian may also prove appropriate because, in contrast to the quantum case, classical states are always separable even when the Hamiltonian is nonseparable (cf. Kronz 1998; Kronz and Tiehen 2002).

4.2. Is Downward Causation Coherent? Among metaphysicians, downward causation has not enjoyed a positive reputation of late. One reason is the suspicion that downward causation entails a violation of the "causal closure of the physical." For example, according to Kim, the causal closure principle (CP) means that "Any physical event that has a cause at time thas a physical cause at t" (1993, p. 280). There are two possible readings of this principle based on the ambiguity carried by the notion of 'physical.' The first, or weaker, reading is the sense of 'physical' as in elements of the physical sciences broadly construed (sometimes encompassing biology as well). On this reading, there are no possible "nonphysical" causes capable of influencing physical systems. The second, or stronger, reading is the sense of 'physical' as in elements of physics. On this reading, there are no causes outside the purview of physics that can influence events in the domain of physics. ${ }^{16}$

Worries about CP normally arise in the context of philosophy of mind, but in the context of Rayleigh-Bénard convection, higher-level physical structures (Bénard cells) constrain and modify the behaviors of the lower-level system constituents (fluid elements). In this case downward causation does not entail any violation of $\mathrm{CP}$ in either a strong or weak sense-nothing outside of physics influences the behavior of the physical system in question. Van Gulick articulates a related worry that if some kind of "system-wide powers" or forces arise at the system level that are not determined by the laws governing the system constituents, then such forces "could override or violate the laws governing the powers of the parts" $(2001,18)$. Such a "violation" could be seen to "threaten the view of the physical world as a closed causal system" (p. 19). Without debating the merits of the metaphysical assumption of the world as a "closed causal system," the constraining/modifying effects Bénard cells have on the motions of fluid elements is an example of a large-scale, nonlocal influence acting on the fluid elements, but this 
influence is consistent with the laws governing the motion of fluid elements, even though these laws and the properties of fluid elements underdetermine the behavior of the cells (recall the degeneracies in the fluid equations). ${ }^{17}$ It is not clear why such large-scale, nonlocal influences should not be viewed as cooperating with the laws governing the system constituents, filling the gaps left by the underdetermination of these forces, as opposed to viewing such influences as somehow overruling these forces. ${ }^{18}$

Downward causation has also been considered as possibly involving an absurdity if it is reflexive; that is to say, if there is a "global-to-local" influence for which the local dynamics provide no sufficient account. In this case there is an apparent absurdity in that downward causation implies "a kind of self-causation, or self-determination" (Kim 1999, 28). Admittedly, self-causation in general is a problematic concept, but the self-causation involved in RayleighBénard convection is of a type where the dynamics of the fluid elements, under particular conditions, give rise to large-scale patterns in the form of Bénard cells. In turn these cells constrain and modify the local dynamics of the fluid elements as the cells form and maintain themselves including managing their own material and energy intake. So Bénard cells arise out of the local dynamics of the fluid elements. But they are also causes in that these cells constrain and modify the dynamics of fluid elements by limiting (allowing) access to states of motion that were previously accessible (inaccessible). Such constraint and maintenance functions of Bénard cells are not absurd in any obvious sense though they appear to act more as formal or structuring causes.

Still, the intelligibility of downward causation has been questioned. Kim has argued that synchronic or simultaneous downward causation is incoherent. ${ }^{19}$ He considers the following situation:

At a certain time $t$, a whole, $W$, has emergent property $M$, where $M$ emerges from the following configuration of conditions: $W$ has a complete decomposition into parts $a_{1}, \ldots$, $a_{\mathrm{n}}$; each $a_{\mathrm{i}}$ has property $P_{\mathrm{i}}$; and relation $R$ holds for the sequence $a_{1}, \ldots, a_{\mathrm{n}}$. For some $a_{\mathrm{j}}$, $W$ 's having $M$ at $t$ causes $a_{\mathrm{j}}$ to have $P_{\mathrm{j}}$ at $t(1999,28)$.

Kim begins his analysis by supposing that $W$ has a particular emergent property $M$, at the given time $t$, and that $M$ 's emergence at $t$ is dependent on a particular micro configuration at $t$, namely that the micro constituents $a_{\mathrm{i}}$ have the particular properties $P_{\mathrm{i}}$. This means, on Kim's analysis, that $W$ has $M$ at $t$ only because each $a_{\mathrm{i}}$ has the particular property $P_{\mathrm{i}}$. Although not explicitly stated, this analysis assumes a general supervenience relation: "causal relations among macroevents and macroproperties must hold in virtue of (and therefore be explicable in terms of) causal relations holding for events and properties at a more basic level. This is only a special case of the general thesis that all the facts in the world supervene on the microphysical facts" (Kim 1993, 358-9). In other words, the macro property $M$ obtains in $W$ only in virtue of the particular microphysical facts (each $a_{\mathrm{i}}$ having the particular property $P_{\mathrm{i}}$ ). According to Kim, "it makes one feel uncomfortable to be told also that $a_{\mathrm{j}}$ is caused to have $P_{\mathrm{j}}$ at the very time, $t$, by the whole's having $M$ at $t$ " (Kim 1999, 28). Kim then seeks to diagnose the source of this "metaphysical discomfort."

It is clear that Kim's discomfort derives from the fact that each $a_{\mathrm{i}}$ having $P_{\mathrm{i}}$ at $t$ is what 
gives rise to $M$, while $M$ is considered to be the cause of each $a_{\mathrm{i}}$ having the particular property $P_{\mathrm{i}}$ at $t$. In other words, the $P_{\mathrm{i}}$ are both cause of and caused by $M$. The claim on which Kim focuses in his diagnosis is that synchronic downward causation involves an incoherence because $W$ having $M$ at $t$ causes $a_{\mathrm{j}}$ to have $P_{\mathrm{j}}$ at $t$, implying that prior to $t, a_{\mathrm{j}}$ does not already have $P_{\mathrm{j}}$ at $t$ (hence, how could $M$ have emerged in the first place). To get the implication, however, one must also invoke what Kim calls the causal-power actuality principle (CPAP):

For an object, $x$, to exercise, at time $t$, the causal/determinative powers it has in virtue of having property $P, x$ must already possess $P$ at $t$. When $x$ is caused to acquire $P$ at $t$, it does not already possess $P$ at $t$ and is not capable of exercising the causal/determinative powers inherent in $P(1999,29)$.

This is a metaphysical principle offered to answer Kim's metaphysical worry. One point to note is that Kim's analysis presupposes the $a_{\mathrm{i}}$ and $W$ are wholly distinct entities. In the case of convection, the fluid elements $\left(a_{\mathrm{i}}\right)$ and the Bénard cells $(W)$ are not wholly distinct entities though the two levels can be distinguished via dynamical time scales $(\S 2.3)$. There is no "complete decomposition into parts," so one key assumption underlying Kim's metaphysical worry does not hold in the case of Rayleigh-Bénard convection.

Another point to note is that because the entities involved are not wholly distinct, the causal relations are not all temporal: the large-scale structures determine/constrain some of the causal properties the fluid elements contribute at the time such contributions are made. One way to see this is via the metaphysical concept of realization. The core idea of realization is that higher-level properties ("macrophysical") are realized by lower-level entities and properties ("microphysical") such that the causal efficacy of the former properties is nothing but the causal efficacy contributed by the latter (e.g., Kim 1998 and 1999; Gillett 2002, pp. 98-101). However, in the case of Rayleigh-Bénard convection, the contributions individual fluid elements make to the Bénard cells is conditioned by the cells: the properties individual fluid elements contribute to the cells are conditioned upon the properties instantiated by the cells. Furthermore, this downward determination is instantaneous; that is, the properties of the fluid elements only contribute when they realize particular other properties such as those associated with the collective motion and large-scale structure of Bénard cells. Hence, CPAP is not applicable to the case of Rayleigh-Bénard convection, nor is there any apparent incoherence involved in this simultaneous upward/downward determination.

The physical picture is the following. Bénard cells emerge out of the local dynamics of fluid elements as a large-scale, nonlocal process with the property that this structure constrains/modifies the states of motion accessible to fluid elements. This is because the Bénard cells act as a control hierarchy. When the cells are established at time $t$, this governing property is established at $t$; it did not exist prior to $t$. Likewise, prior to $t$, the trajectories of fluid elements had the property of accessing various states of motion, a property they loose at $t$ due to the largescale, nonlocal governing effects of Bénard cells. The causal constraints/modifications on the motion of the fluid elements in this case is synchronic: the emergence of the self-regulating largescale pattern is simultaneous with the modifications of/constraints on the accessible states of motion. If it were not synchronic, the pattern would not arise. ${ }^{20}$ Because of the long-range 
correlations, an individual fluid element can only execute motions allowed to it by all other fluid elements (e.g., all the Bénard cells will determine whether or not a particular fluid element will stay in formation with its current cell or migrate to a neighboring cell).

On Kim's picture, the Bénard cells (or the ever-growing pattern) can have no causal influence at time $t$ on the fluid elements. Any influence large-scale structures have on fluid elements can only occur at some delay $t+\tau$, where $\tau$ is some small positive number (set aside the fact that on his view the constraining influence of the cells must be a chimera). This would be the physical import of CPAP, the idea being that the fluid elements contribute all the casual properties had by the large-scale structures, hence the need for whatever causal influences the latter have to lag the contributions of the fluid elements. However, this misses out the notion of control hierarchy at work here, namely the nonlocal constraining function the (developing) largescale structures exert on the fluid elements. As the patterns form, they exhibit a downward determinative influence on the fluid elements, making the contribution of the latter at $t$ to the large-scale conditional on the pattern at $t$. That is to say, large-scale structures arise out of fluid elements, but they also condition or constrain the contributions the fluid elements can make, namely by modifying or selecting which states of motion are accessible to the fluid elements (e.g., due to enslavement; see Haken 1983b). Hence, if there was no synchronic relationship between the constraints and the fluid elements, there would be no pattern. There are no "new forces" coming out of nowhere; rather, the fluid is governing itself in a complex set of interactions among parts and wholes.

4.3 Comparison with Sperry Cases. Is the candidate for downward causation proposed here, Rayleigh-Bénard convection, different from Sperry cases? In his example of the wheel rolling down a hill, the structural properties of the wheel supposedly constrain the movement of the molecules composing the wheel, carrying them down the hill. Kim has argued $(1999,29-31)$ that such cases as the motion of the whole wheel can be analyzed as the summed motion of parts ${ }^{21}$ (and, hence, is a diachronic effect). In contrast, the kind of governing influence, which Bénard cells have on the fluid elements, cannot be so analyzed (it is synchronic).

Perhaps the constraining/modifying behavior of the Bénard cells is merely an artifact of our explanation like that of Sperry's wheel (hence, only epistemological at best), or perhaps the ascription of ontological status to downward causation in Rayleigh-Bénard convection is misguided because such large-scale, nonlocal processes are not really causal since all 'proper' causation in physical systems has to be local, efficient causation (e.g., as is often assumed to be the case in analyzing examples like Sperry's wheel). Although space does not permit a full response, such objections overlook the close interplay between experiment and theory regarding the contextual factors involved in convection (e.g., symmetries, long-range forces, spatial correlations involving all fluid elements, dynamical history) and also beg the question about how such large-scale, nonlocal structuring factors operate.

One might respond that although no reductive analysis of these effects can be given currently, some future better theory will be able to explain, from the point of view of the fluid elements, the necessary and sufficient conditions for the behavior of the cells (hence, blocking epistemological emergence and, presumably, ontological emergence as well ${ }^{22}$ ). Such a reply presupposes metaphysical reductionism, but there is not much evidence for this as a general 
assumption, at least not in any of our sciences (Bishop 2006a; 2006b; Bishop and Atmanspacher submitted). On the other hand, there are a number of cases, for example in chemistry and thermodynamics, where reductionism of this type fails (Bishop 2004; 2006b; Bishop and Atmanspacher submitted). After all, the fact that a system is composed of constituents does not imply that all the system's behaviors are caused by the behaviors of its constituents in a resultant fashion (that is to confuse analysis of systems with reductionism of systems to their components). To presume that a future better theory will be reductionist is simply to beg the question.

Furthermore, although there is a microscopic approach to the fundamental conserved quantities of fluid systems (e.g., particle number, energy and momentum) analogous to the hydrodynamic approach discussed in $\S 3$, this kinetic approach still makes irremovable references to such nonlocal and system-wide features as conservation laws, symmetries and dynamical modes of the system as a whole in order to understand the behavior of a test particle (Pomeau and Résibois 1975). ${ }^{23}$ Collective features of the system still play an ineliminable, crucial determinating role in the dynamics of system constituents even from the kinetic perspective.

\section{Concluding Remarks.}

There are several lines of evidence, including molecular dynamics simulations aimed at giving a microscopic view of fluid dynamics, indicating that the hydrodynamic description is valid, even at molecular length and time scales (Mareshal 1997, 317-20). This is to say, the hydrodynamic description of fluids is not valid because it "averages over" individual interactions of fluid elements, a misleading characterization ignoring the role nonlocal and system-wide features play in the derivation, closure and solution of the fluid equations. Rather, the description is valid because it includes effects that are absent from considering just the local (nearest-neighbor) interactions of individual fluid elements. For instance, hydrodynamics includes the evolving relations that develop among all fluid elements with each other and their constraining influence on the dynamics of individual fluid elements and nearest-neighbor interactions.

Rayleigh-Bénard convection, then, looks to be a promising candidate for studying interlevel causation, flowing from the fluid elements upward and from the large-scale structures downward. The tight integration between the effects of the dynamics in these different hierarchies-representing different length and time scales-with the Bénard cells acting as a control hierarchy, can be an important source of insight for enriching and extending our understanding of causation. For example, typical philosophical accounts of causation-e.g., counterfactual, logical, probabilistic, process, regularity, structural-were developed within the context of a "linear worldview" heavily influenced by a nearly exclusive focus on linear models of Newtonian mechanics. In the context of nonlinear systems, such as convection, there are many additional channels of interaction not envisioned in such a linear framework (e.g., open systems exchanging matter and energy with their environments (a form of situatedness), nonlinear feedback loops, inter-level interactions, self-organization, whole-part relations, memory effects). Dealing with these additional forms of interaction appears difficult without modifying or extending typical causal accounts. For instance, process theories lack an adequate account of the structural influence of large-scale, global influences on parts, while structural theories lack an adequate account of large-scale nonlocal processes. Counterfactual approaches maintain 
(roughly) that a cause is some event in the absence of which the effect would not occur; the applicability of this approach, however, is substantially limited by the multiple realizability exhibited by many of the nonlinear behaviors in complex systems (regularity accounts suffer similar limitations).

In particular, as proposed in this essay, fluid convection can serve as a model for further study of causal accounts. More to the point, such a model is well suited for exploring emergence and downward constraint, clearly going beyond Sperry's examples in these respects. Furthermore, convection processes can serve to test the applicability and generality of many of the assumptions and arguments invoked in philosophical discussions of reduction, emergence and downward causation (e.g., Kim's CPAP). Getting clearer about the applicability of such assumptions as well as about emergence and downward causation in the context of concrete examples like convection might also shed light on how to think about these concepts in terms of cognitive function and broader issues in philosophy of mind. For example, TV was originally proposed in the context of an embodied cognitive science, where consciousness might be viewed as an emergent feature of the nonlinear neural dynamics constrained and modified by its presence in a body ecologically situated in a wider environment (Thompson and Varela 2001). Sharpening our understanding of emergence and downward determination using concrete examples from nonlinear dynamics-with all the features listed in \$2.2-should prove useful in understanding and evaluating the evidence for the downward determination of mental activities on, for example, the dynamics of epileptic activity (e.g., changes in the patterns of the neural dynamics; Le Van Quyen et al. 1997a, b; Thompson and Varela 2001).

So it appears that classical physics still holds many philosophical lessons for us to learn.

\section{Acknowledgments}

An earlier version of this essay was presented at the 2002 BSPS Annual Meeting in Glasgow, Scotland. The essay also has benefitted greatly from discussions with Harald Atmanspacher, Fred Kronz, Michael Silberstein and Evan Thompson. Much of the research for this project was carried out while on a post-doc in the Abteilung für Theorie und Datenanalyse, Institut für Grenzgebiete der Psychologie, Wilhelmstrasse 3a D-79098 Freiburg, Germany.

\section{References}

Alder, B. and Wainwright, T. (1970). Decay of the velocity autocorrelation function. Physical Review A, 1, 18-21.

Auyang, S. (1998). Foundations of complex-system theories: in economics, evolutionary biology, and statistical physics. (Cambridge: Cambridge University Press.)

Batchelor, G. (1967). An introduction to fluid dynamics. (Cambridge: Cambridge University Press.)

Batterman, R. (1993). Defining chaos. Philosophy of Science, 60, 43-66.

Bishop, R. C. (2004). Nonequilibrium statistical mechanics Brussels-Austin style. Studies in History and Philosophy of Modern Physics, 35, 1-30. 
Bishop, R. C. (2006a). The hidden premise in the causal argument for physicalism. Analysis, 66, 44-52.

Bishop, R. C. (2006b). Patching physics and chemistry together. Philosophy of Science, 72, forthcoming.

Bishop, R. C. and Atmanspacher, H. (Submitted). Contextual emergence in the description of Properties.

Bishop, R. C. and Kronz, F. K. (1999). Is chaos indeterministic? (In M. Dalla Chiara, G. Roberto and F. Laudisa (Eds.), Language, Quantum, Music: Selected Contributed Papers of the Tenth International Congress of Logic, Methodology \& Philosophy of Science, Florence, August 1995 (pp. 129-41). London: Kluwer Academic Publishers.)

Brand, M. (1980). Simultaneous causation. (In P. van Inwagen (Ed.), Time and Cause (pp. 137153). Dordrecht: D. Reidel Publishing.)

Busse, F. (1978). Non-linear properties of thermal convection. Reports on Progress in Physics, 41, 1929-1967.

Cross, M. and Hohenberg, P. (1993). Pattern formation outside of equilibrium. Reviews of Modern Physics, 65, 851-1112.

Crutchfield, J. (1994). Observing complexity and the complexity of observation. (In H.

Atmanspacher and G. Dalenoort (Eds.), Inside Versus Outside (pp. 235-72). Berlin: Springer-

Verlag.)

Dirac, P. (1949). Forms of relativistic dynamics. Reviews of Modern Physics, 21, 392-399.

Earman, J. (1986). A Primer on Determinism. (Dordrecht, The Netherlands: D. Reidel

Publishing.)

Gillett, C. (2002). The varieties of emergence: their purposes, obligations and importance. Grazer Philosophische Studien, 65, 95-121.

Grassberger, P. (1989). Problems in quantifying self-generated complexity. Helvetica Physica Acta, 62, 489-511.

Greenside, H., Coughran, Jr., W., Schryer, N. (1982). Nonlinear pattern formation near the onset of Rayleigh-Bénard convection. Physical Review Letters, 49, 726-729.

Guyon, E., Hulin, J.-P., Petit, L. and Mitescu, C. (2001). Physical Hydrodynamics. (Oxford:

Oxford University Press.)

Hacking, I. (1983). Representing and Intervening: Introductory Topics in the Philosophy of

Science. (Cambridge: Cambridge University Press.)

Hacking, I. (1984). Experimentation and scientific realism. (In J. Leplin (Ed.), Scientific Realism (pp. 154-172). Berkeley: University of California Press.)

Haken, H. (1983a). Synergetics: An Introduction, Third Revised and Enlarged Edition. (Berlin: Springer-Verlag.)

Haken, H. (1983b). Advanced Synergetics: Instability Hierarchies of Self-Organizing Systems and Devices. (Berlin: Springer-Verlag.)

Huemer, M. and Kovitz, B. (2003). Causation as simultaneous and continuous. The Philosophical Quarterly, 53, 556-565.

Hill, R. (1967). Instantaneous action-at-a-distance in classical relativistic mechanics. Journal of Mathematical Physics, 8, 201-220.

Hobbs, J. (1991). Chaos and indeterminism. Canadian Journal of Philosophy, 21, 141-164. 
Juarrero, A. (1999). Dynamics in Action: Intentional Behavior As a Complex System. (Cambridge, MA: MIT Press.)

Kellert, S. (1993). In the Wake of Chaos. (Chicago: University of Chicago Press.)

Kim, J. (1993). Supervenience and Mind. (Cambridge: Cambridge University Press.)

Kim, J. (1998). Mind in a Physical World: An Essay on the Mind-Body Problem and Mental Causation. (Cambridge, MA: MIT Press.)

Kim, J. (1999). Making sense of emergence. Philosophical Studies, 95, 3-36.

Kronz, F. (1998). Nonseparability and quantum chaos. Philosophy of Science, 65, 50-75.

Kronz, F. and Tiehen, J. (2002). Emergence and quantum mechanics. Philosophy of Science, 6,: 324-347.

Le Van Quyen, M. (1997a). Temporal patterns in human epileptic activity are modulated by perceptual discriminations. NeuroReport, 8, 1703-1710.

Le Van Quyen, M. (1997b). Unstable periodic orbits in human epileptic activity. Physical Review E, 56, 3401-3411.

Malraison, B., Atten, P., Bergé, P. and Dubois, M. (1983). Dimension of strange attractors: an experimental determination for the chaotic regime of two convective systems," Journal of Physics Letters, 44, 897-902.

Mareschal, M. (1997). Microscopic simulations of complex flows. Advances in Chemical Physics, 100, 317-392.

McLaughlin, B. (1982). British emergentism. (In A. Beckermann, H. Flohr and J. Kim (Eds.), Emergence or Reduction? Essays on the Prospects of Nonreductive Physicalism (pp. 49-93).

Berlin: Walter de Gruyter.)

Pathria, R.(1972). Statistical Mechanics. (Oxford: Pergamon Press).

Pattee, H. (1973).“The physical basis and origin of hierarchical control. (In H. Pattee (Ed.), Hierarchy Theory: The Challenge of Complex Systems (pp. 69-108). New York: George Braziller.)

Paul, M., Chiam, K.-H., Cross, M. and Greenside, H. (2003). Pattern formation and dynamics in Rayleigh-Bénard convection: numerical simulations of experimentally realistic geometries. Physica D, 184, 114-126.

Pomeau, Y. and Résibois, P. (1975). Time dependent correlation functions and mode-mode coupling theories. Physics Reports, 19, 63-139.

Primas, H. (1983). Chemistry, Quantum Mechanics and Reductionism: Perspectives in Theoretical Chemistry. (Berlin: Springer-Verlag.)

Scott, A. (1999). Nonlinear Science: Emergence \& Dynamics of Coherent Structures. (Oxford: Oxford University Press.)

Silberstein, M. and McGeever, J. (1999). The search for ontological emergence. The Philosophical Quarterly, 49, 182-200.

Smith, P. (1998). Explaining Chaos. (Cambridge: Cambridge University Press.)

Sperry, R. (1969). A modified concept of consciousness. Psychological Review, 76. 532-536.

Stone, M. (1989). Chaos, prediction and laplacean determinism. American Philosophical

Quarterly, 26, 123-31.

Taylor, R. (1963). Causation. Monist, 47, 287-313.

Taylor, R. (1966). Action and Purpose. (Englewood Cliffs, NJ: Prentice-Hall.) 
Teller, P. (1986). Relational holism and quantum mechanics. British Journal for the Philosophy of Science, 37, 71-81.

Terry, P. (2000). Suppression of turbulence and transport by sheared flow. Reviews of Modern Physics, 72, 109-165.

Thompson, E. and Varela, F. (2001). Radical embodiment: neural dynamics and consciousness. TRENDS in Cognitive Science, 5, 418-425.

Van Gulick (2001). Reduction, emergence and other recent options on the mind/body problem: a philosophic overview. Journal of Consciousness Studies, 8, 1-34.

Wackerbauer, R., Witt, A., Atmanspacher, A., Kurths, J. and Scheingraber, H. (1994). A comparative classification of complexity measures. Chaos, Solitons, Fractals, 4, 133-173.

Walter, H. (2001). Neurophilosophy of Free Will: From Libertarian Illusions to a Concept of Natural Autonomy, C. Klohr (Trans.). (Cambridge, MA: MIT Press.) 


\section{Endnotes}

1.See McLaughlin 1982 for a discussion of the origin and history of the terms 'resultant' and 'emergent.'

2.A nonlocal description in nonlinear dynamics refers to a description that necessarily must refer to system-wide and environmental features in addition to local interactions of individual with one another.

3.Physical variables are required to define the state space over which probability measures are defined. Thus, they are implicitly involved in probabilistic definitions.

4.Note that typically fundamental forces (e.g., gravity, electromagnetism) are not explicitly identified with constraints.

5.For a fluid at rest, there are two interesting extreme cases. When the mass of the given fluid is much smaller than that of the surrounding matter, the external gravitational field is approximately uniform over the entire region occupied by the fluid (depending on the surrounding matter distribution). When the mass of the given fluid is large and isolated from other matter (e.g., a gaseous star), the volume force on a particular fluid element is due to the gravitational attraction of all other fluid elements. In the former case, gravity contributes to the global external constraints on the motions of fluid elements, while in the latter case, gravity generated by the fluid itself is the main nonlocal internal constraint on such motions.

6.For a summary of other possible correlation effects in nonlinear dynamical systems, see Bishop (2004).

7.Typically the Reynolds number is used to characterize behavior in convection systems. Since this parameter is proportional to $\Delta T$, I have chosen to focus on the temperature gradient as this is more physically perspicuous for the current description.

8.The growth rate of instabilities depends only on the wave number; that is, although the fluctuations in combination with the system geometry and symmetries lead to a selection of one growing mode, its growth rate is determined solely by global system properties (Cross and Hohenberg 1993, §§VI and VIII; Haken 1983b, chapter 7). For Rayleigh-Bénard convection, the most unstable mode scales as the distance of plate separation.

9. Some have complained that these reductions are ultimately epistemological in the sense that they are simplified descriptions of physical systems in terms of a reduced number of variables so that these variables should not be considered as causal (e.g., Walter 2001, pg. 177 and references therein). However, this complaint ignores the experimental support for the causal role of these variables (e.g., Malraison et al. 1983). One could justify the causal effectiveness of such variables along the lines of entity realism (e.g., Hacking 1983; 1984): if an entity can be used in manipulations and interventions, then it should count experimentally as real. The reduced variables in question fulfil the appropriate causal roles. 
10.Each fluid element is correlated with all other elements. When considering an idealized fluid system of infinite extent, the correlation length would become infinite. However, given a finite geometry and boundaries, the correlation length is the length of the largest dimension of the system.

11.That is, the overall motion of a large volume of fluid.

12.For mathematical and experimental details, along with other examples of similar processes, see (Terry 2000).

13.See (Bishop 2004) for examples in nonequilibrium statistical mechanics, where collective effects not reducible to local interactions contribute to the dynamics of systems.

14. The bulk flow of fluids also plays an important role in the formation and dynamics of patterns by contributing nonlocal effects (e.g., acting over many roll widths in Rayleigh-Bénard convection; see Paul et al. 2003). Bulk flow of the entire convecting system gives rise to an additional slow time dependence as it advects the pattern, which can contribute to pattern stability, for example.

15.These considerations lie at the heart of self-organization and pattern formation. Given the same dynamics, changes in experimental setup can favor the formation of one form of Bénard cell over others. Due to sensitive dependence, given identical experimental setups, fluctuations at the level of fluid elements can lead to different pattern formation on multiple runs of an experiment (e.g., the direction of rotation of cells). The dynamics makes possible a number of different ways in which the fluid elements can organize themselves into coherent large-scale patterns (i.e., a number of different ways in which the nonlocal relations among fluid elements can arise).

16.I have argued elsewhere (Bishop 2006a) that this latter reading of 'physical' renders CP invalid and question begging.

17.This amounts to a denial of one of the core principles of atomistic physicalism as identified by van Gulick: "The only law-like regularities needed for the determination of macro features by micro features are those that govern the interactions of those micro features in all contexts, systemic or otherwise" (2001, pg. 18). However, the degeneracies in fluid motion make it clear that specific global and/or system-wide contexts (e.g., symmetries and boundaries, collective effects, history of development) are crucial to determining fluid behavior in convection; hence, the denial of this principle is well justified in the case of fluids because the contributions that the laws and microfeatures make to Bénard cells are conditioned by context.

18.This latter possibility is a metaphysician's worry if CP is taken in the strong sense, i.e., that physics is causally closed. But there is every reason to think that the weak construal of CP is more reasonable given that physics-as with all sciences-is highly idealized and given that there appear to be clear examples where chemical and biological structures constrain or modify the behavior of electrons, molecules and such (Bishop 2006a). That is, these structures fill the 
underdetermination left by the fundamental forces of physics via context (e.g., Bishop 2006b; Bishop and Atmanspacher submitted).

19.Kim notes that this situation "involves the controversial idea of simultaneous causation (where a cause and its effect occur at the same time)" (1999, 36, fn. 34). I take it that Kim's worry about simultaneous causation is whether it can be made metaphysically coherent, not physically coherent. There are physically coherent expressions of synchronic effects in physics as in relativistically consistent instantaneous action-at-a-distance theories (Dirac 1949; Hill 1967). Regarding the latter, there is an empirical question whether such simultaneous influences exist, but they are physically intelligible. See Taylor (1963; 1966), Brand (1980), and Huemer and Kovitz (2003) for defenses of the metaphysical coherence of simultaneous causation.

20.This is somewhat oversimplified. In reality Bénard cells form over some finite, but short (at least for small systems) time period. However, at each instant during this period of pattern formation, the corresponding large-scale, nonlocal constraints appear modifying the accessible states of fluid element motion. Downward constraint or determination is still synchronic as the large-scale, nonlocal processes alter the states of motion accessible by the fluid elements instant by instant.

21.Presumably there is a limit to how far down such a reductive analysis can go before it becomes nonsensical, but this has to do with the transition from classical mechanics to quantum mechanics.

22.See van Gulick (2001) for a lucid discussion of these distinctions.

23. Note that even the definitions of conservation laws for local quantities in a fluid make irremovable references to system-wide properties (e.g., Guyon et al. 2001, chapters 3 and 5). 